\title{
RNN-based forecasting of indoor temperature in a naturally ventilated residential building
}

\author{
Kui Weng ${ }^{1}$, Monjur Mourshed ${ }^{1}$ \\ ${ }^{1}$ School of Engineering, Cardiff University, Cardiff CF24 3AA, United Kingdom
}

\begin{abstract}
Natural ventilation is an effective passive control strategy to improve building energy efficiency, indoor thermal comfort and air quality. Near real-time model-based control of window openings for natural ventilation requires forecasts completed within a short time, typically seconds. However, widely-used physics-based simulation is time-consuming and entails high computation cost. This study is aimed at developing a Recurrent Neural Network (RNN) model for forecasting indoor temperature using seasonal window opening schedules and ambient conditions. The data-driven forecasting approach utilizes simulated indoor dry-bulb temperature (DBT) and relative humidity $(\mathrm{RH})$ based on ambient parameters (DBT, RH, and wind speed and direction), time (time of day, day of year), building thermal parameters and window characteristics (location, opening size and type) to train the RNN model. The results show that the proposed RNN algorithm is effective in predicting indoor environmental conditions with considerable accuracy $\left(\mathrm{R}^{2}\right.$ $=0.956)$ and outperforms statistical methods by at least $20 \%$ in the same measure. Window opening area is highly correlated to the hourly change of indoor temperature. Prediction errors for indoor operative temperature are less than $1{ }^{\circ} \mathrm{C}$ for $70 \%$ of the time and less than $2^{\circ} \mathrm{C}$ for $93 \%$ of the time. The speed and accuracy of a trained network illustrate the potential of the method for near-real time control of buildings and systems while maintaining occupant thermal comfort.
\end{abstract}

\section{Introduction}

Anthropogenic greenhouse gas (GHG) emissions are the key driver for observed global climate change (Stocker, 2014), resulting in increased ambient temperature and summertime overheating risk in buildings (Mourshed, 2011). Globally, $40 \%$ of the total energy and $30 \%$ of the total $\mathrm{CO}_{2}$ emissions are attributed to the building sector (Ahmad, Mourshed, Mundow, Sisinni, \& Rezgui, 2016). In Europe, buildings account for $40 \%$ of the total energy use and $36 \%$ of the greenhouse gas emissions (Grozinger, Boermans, Wehringer, \& Seehusen, 2014). Most of the carbon emissions from buildings come from their operation. In 2014, the operation of the buildings accounted for three-quarters of the UK emissions (Giesekam \& Pomponi, 2018). Therefore, improving the operational efficiency plays an important role in mitigating the impacts of climate change. On the other hand, with the rising ambient temperature due to anthropogenic climate change, there is an increasing need for cooling, leading to an increase in electricity usage (Ramponi, Angelotti, \& Blocken, 2014).

Driven by thermal buoyancy or the wind, natural ventilation is a key sustainable strategy for improving air quality, maintaining thermal comfort and reducing energy use in buildings (Tong, Chen, Malkawi, Liu, \& Freeman, 2016). Natural ventilation allows air exchange between the interior and exterior without the aid of mechanical means, which results in saved energy. Natural ventilation can be adopted as a standalone strategy to meet fresh air requirements and to cool down a building, or it can be used as a low-carbon strategy to supplement mechanical cooling and ventilation in mixed-mode operation to minimize energy demand (Weng, Meng, \& Mourshed, 2018). Compared with mechanical ventilation and airconditioning (AC), natural ventilation has the potential to significantly save cooling energy consumption and reduce carbon emissions (Schulze, Gurlich, \& Eicker, 2018) while allowing occupants to adapt the immediate environment to their thermal comfort preferences. In the $\mathrm{AC}$ and mechanically ventilated buildings, space heating and cooling through fans and pumps by moving air or refrigerant accounted for up to $50 \%$ of the total carbon emissions (Lomas, 2007). Night ventilation has been found to save $35 \%$ of the cooling energy consumption in a ventilated photovoltaic double-skin facade building in Berkeley, USA (Peng, et al., 2016). In northern Europe (including the British Isles), passive cooling of buildings via night ventilation shows high cooling potential (120$180 \mathrm{Kh}$ ) in the hottest months of the year (Artmann, Manz, \& Heiselberg, 2007).

Several challenges are associated with the adoption of natural ventilation as a low-carbon strategy. First, occupant thermal comfort needs to be maintained at an optimal level, especially during the cold winter and intermediate spring and autumn seasons when ambient conditions are lower than the optimum in cold and temperate climates. In summer months, and especially in hot and arid climates, ventilation heat gain may be counterproductive to indoor thermal comfort. The consideration of comfort; i.e., the indoor conditions as a decision constraint requires building management systems to predict the parameters, typically at the subhourly control timestep. Second, predicting indoor temperature using network airflow or computational fluid dynamics (CFD) simulation is a time- and computeintensive process which may greatly affect their adoption 
in real-time or near real-time applications. Hence, the time- and resource-efficient prediction of indoor conditions in a naturally ventilated system is still an open research issue (Chenari, Carrilho, \& da Silva, 2016), especially for model-based control of openings for natural and mixed-mode ventilation.

Reinforcement Learning (RL), a branch of artificial intelligence, is of increasing interest to the built environment community for applications in energy conservation and thermal comfort in buildings. The RL method is advantageous over heuristic and physics-based modelling as a response generator - demonstrating its potential for use in model-predictive control (MPC) to find optimized control parameters and schedules without explicitly modelling the underlying system. Chen et al. (2018) used Q-learning to optimize the control of HVAC and window systems to minimize thermal discomfort and energy load. Markovic et al. (2019) presented a deep neural network to predict window states by training measured weather (outdoor temperature, humidity, wind speed, wind direction, global diffuse radiation, average pressure) and indoor conditions (indoor temperature, humidity, $\mathrm{CO}_{2}$, presence, thermostat set point, window state) data. Wang et al. (2017) employed a long-short term memory (LSTM) recurrent neural network (RNN) for HVAC control, improving the energy efficiency by $2.5 \%$ and thermal comfort by $15 \%$ on average, compared to the traditional on/off control.

Deep learning algorithms such as RNNs and neural sequence models have been widely applied in non-linear time series prediction. Because of the presence of feedback connections, it does not require the past time series inputs as it can establish recurrent connections between a final output and events in its structure. RNN has been widely used in forecasting energy consumption in buildings (Ruiz, Cuellar, Calvo-Flores, \& Jimenez, 2016). However, the application of deep learning for forecasting building indoor environmental conditions (e.g., indoor temperature) is relatively new and of increasing interest in the recent years. The parameters used in the deep learning for forecasting indoor temperature often include local weather (DBT, RH, wind speed) and indoor environment conditions (building characteristics, schedules for the sub-systems such as windows) (Xu, Chen, Wang, Guo, \& Yuan, 2019). Those parameters have also been widely used in neural network forecasting models to predict indoor temperature (Marvuglia, Messineo, \& Nicolosi, 2014).

In this work, an LSTM-RNN based machine learning algorithm has been developed to predict indoor operative temperature in a residential building.

\section{Methodology}

An LSTM-RNN model was trained to forecast the indoor operative temperature in a residential building. The dataset used for training and testing was based on the results from a whole-building simulation of a naturally ventilated building, where various window opening areas in the living room were parametrically simulated.

\section{Building model}

The climate in the UK is temperate and oceanic. However, relatively warm summer is common in the South such as in London and Cardiff. In addition, most of the residential buildings have no mechanical cooling but use only natural ventilation through window opening. Therefore, it is worth investigating how natural ventilation through the opening of windows influences indoor temperatures in residential buildings in Cardiff.

EnergyPlus was used to model a two-floor terraced house located in Cardiff, UK, as shown in Figure 1. The building shares its two side walls with the neighboring buildings. Built in 2014, the construction conforms to the recent UK building regulations, with double glazing and highly insulated external walls. The living room in the ground floor was used to assess the effects of window opening on indoor temperature. The hourly and weekly ventilation schedules; i.e., window opening area, considered the occupancy patterns during weekdays and weekends. The window of the living room faced North and opening areas ranging from $0 \%$ to $100 \%$ of the window size were simulated parametrically. Windows were assumed to remain open for longer during weekends as occupants typically stay at home. Current Cardiff weather data was obtained from UK Climate Projections (UKCP) and used as an input to the model.

\section{RNN model}

As a data-driven approach, RNN does not require the creation of a physics-based model to simulate the underlying system but use historical data. However, the training of the RNN involves the adjustment of the neural network to improve prediction accuracy. The hyperparameters (e.g., learning rate) were determined using a trial-and-error method. The network of the RNN model in this research includes two hidden layer units, a learning rate $(l r)$ of 0.001 , the batch size of 64 and an epoch of 100 .

\section{Dataset}

Table 1 illustrates the inputs and outputs of the LSTMRNN model for forecasting indoor operative temperatures. The hourly operative temperature of the living room, generated from the simulation of the building model was utilized as the RNN output variable. To consider the impact of natural ventilation on indoor temperature, window opening area was varied between $0 \%$ and $100 \%$ at a $10 \%$ interval.

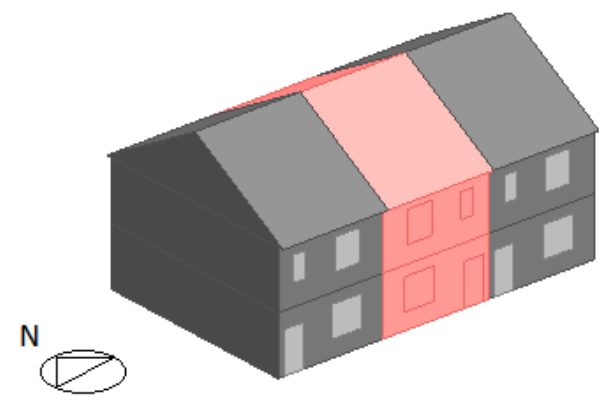

Figure 1: The geometry of two-floor terraced house. The $3 D$ model shows the front facade, floors and pitched roof of the building. 
Table 1: Variables in the LSTM-RNN model

\begin{tabular}{|c|c|}
\hline Name & Type \\
\hline Indoor operative temperature $\left({ }^{\circ} \mathrm{C}\right)$ & Output \\
\hline Month & Input \\
\hline Day & Input \\
\hline Hour & Input \\
\hline Day of week & Input \\
\hline Outdoor air temperature $\left({ }^{\circ} \mathrm{C}\right)$ & Input \\
\hline Direct solar radiation $\left(\mathrm{W} / \mathrm{m}^{2}\right)$ & Input \\
\hline Diffuse solar radiation $\left(\mathrm{W} / \mathrm{m}^{2}\right)$ & Input \\
\hline Wind speed $\left(\mathrm{m}^{2} / \mathrm{s}\right)$ & Input \\
\hline Outdoor relative humidity $(\%)$ & Input \\
\hline Window opening area ratio $(\%)$ & Input \\
\hline
\end{tabular}

Each opening strategy was modelled for one-year that generated 8760 rows of hourly data. The eleven window opening strategies generated a dataset comprising 96360 (8760 hours $\times 11$ window opening areas) hours, which were used for training, validation and testing of the RNN model. The weather data (e.g., outdoor temperature) and window opening area were used as inputs to the model. In addition, time, including month, day and hour, and the day of week ( 1 to 7 for Monday to Sunday) were adopted as the inputs considering the effect of time on weather and hourly indoor temperature.

\section{Training and testing}

To retain the performance of training in a deep learning neural network, a proper split of data is needed for training, validation and testing. This research adopted $6 / 2 / 2$, which is the most often used data splitting method. The dataset is divided into three parts: training (60\%), validation (20\%) and testing (20\%). Training and validation were used to optimize the training model for testing. Without validation, the model could be overfitted or underfitted, which could lead to a bad generalization.

Figure 2 illustrates the results from hyper-parameter tuning. Hyper-parameters such as the learning rate and epoch are important for forecasting performance. Learning rate controls the amount of change on adjusting the weights of the network regarding the loss gradient. In this model, different learning rates were chosen during training: $0.1,0.01,0.001$ and 0.0001 . Training and validation loss were used to evaluate the performance (against time and accuracy) of the model to identify appropriate hyper-parameters.

As shown in Figure 2b, $2 \mathrm{c}$ and $2 \mathrm{~d}$, both the training loss and validation loss decreased as the epoch increased. Figure 2a showed that the learning rate of 0.1 was too large and underfitted the model, which could easily miss the global or local minima in gradient descent. Figure $2 b$ and $2 \mathrm{c}$ fitted the data better compared to $2 \mathrm{a}$ and $2 \mathrm{~d}$. Especially in 2c, both training and validation loss decreased gradually and fitted closely after 100 epochs. Figure $2 \mathrm{~d}$ indicated underfitting before 200 epochs. More epochs were needed to improve the forecasting performance.

After training and validation, the saved model was applied on the testing dataset. In this part, actual indoor temperatures were compared to the predicted indoor temperatures to assess the forecasting performance.
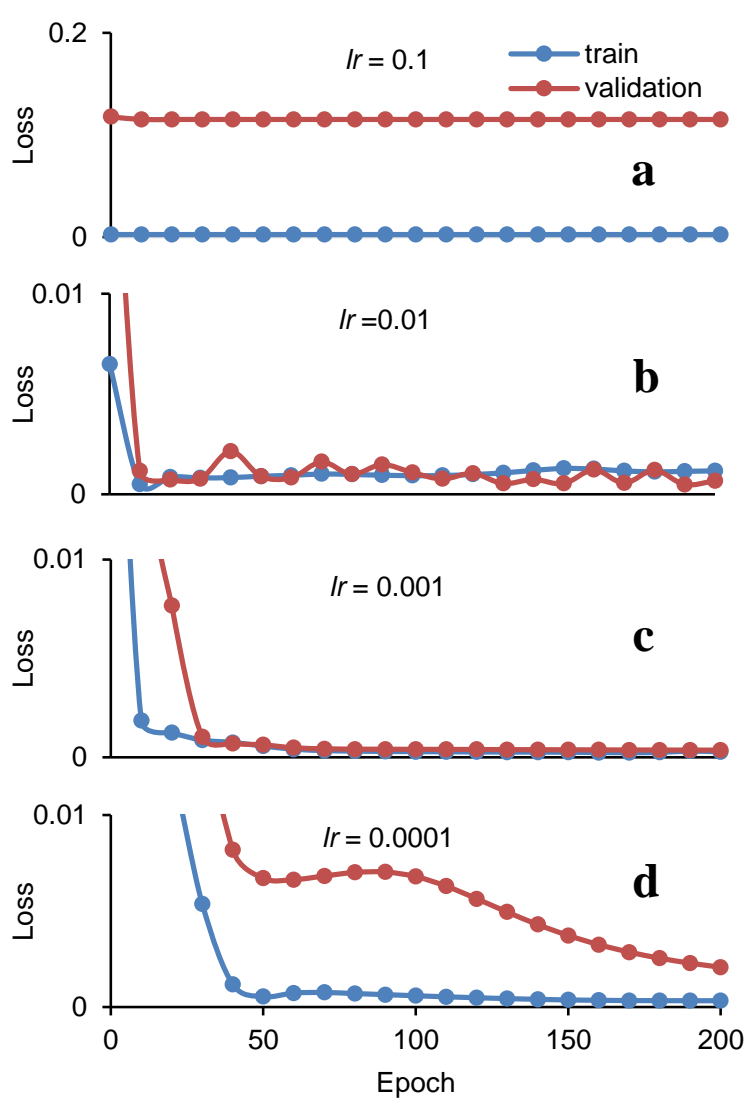

Figure 2: Model training and validation loss based on various learning rate.

Table 2: Summary of hyper-parameter tuning results

\begin{tabular}{|c|c|c|c|c|c|c|}
\hline $\mathbf{M}$ & $\boldsymbol{l} \boldsymbol{r}$ & Epoch & $\mathbf{R}^{\mathbf{2}}$ & MAE & RMSE & MAPE \\
\hline 1 & 0.01 & 200 & 0.937 & 1.051 & 1.497 & 14.949 \\
\hline 2 & 0.001 & 200 & 0.931 & 1.204 & 1.574 & 17.978 \\
\hline 3 & 0.001 & 100 & 0.956 & 0.944 & 1.262 & 20.601 \\
\hline 4 & 0.0001 & 200 & 0.924 & 1.262 & 1.645 & 17.967 \\
\hline 5 & 0.0001 & 1000 & 0.956 & 0.898 & 1.252 & 20.653 \\
\hline
\end{tabular}

Note: $\mathrm{M}=$ Model

To assess the performance of the LSTM-RNN model, determination coefficient $\left(\mathrm{R}^{2}\right)$, mean squared error (MSE), root mean square error (RMSE) and mean absolute percent error (MAPE) were calculated for each iteration of the RNN model.

Forecasting accuracy was analysed through comparing the prediction results and the actual values. Table 2 summarises forecasting accuracy summary for models based on different learning rates and epochs. As shown in Figure 2c, the model would fit the data well after 100 epochs. Overfitting problem was possible with 200 epochs. Therefore, model 4 with the learning rate of 0.001 and 100 epochs was selected for retraining. Compared to model 2, the forecasting accuracy was improved from 0.931 to 0.956 in model 4 . The reduction of training epochs showed a significant effect on mitigating the overfitting in model 4 . Training with smaller learning rate such as 0.0001 and more epochs (e.g., 1000) could achieve higher forecasting accuracy in model 5 . However, 
it took longer for training, and accuracy did not improve much compared to model 3.

\section{Results and discussion}

The trained RNN model was used to forecast the indoor operative temperatures in the residential building. After training and validation, the weights of each inputs were calculated, through which the related output, indoor temperature, was produced. Figure 3 compares the forecasted results and the actual indoor operative temperatures over a random 100 hours. The result showed an overall reliable performance in forecasting the indoor temperatures using the RNN method. There are 73 hours out of 100 hours of predicted indoor temperatures with an error of less than $1{ }^{\circ} \mathrm{C}$. The maximum difference between the forecasted and actual temperature was $2.36^{\circ} \mathrm{C}$, while the minimum was 0.01. In addition, the model could predict the peaks of indoor temperatures over the hours, which was important for indoor thermal comfort and overheating risk assessment. Especially in the summer, the high or peak indoor temperatures in naturally ventilated dwellings can result in overheating, which is a threat to the health and well-being of occupants.

Figure 4 illustrates the difference between the forecasted and actual indoor operative temperatures for around 20,000 hours. It demonstrates the errors; i.e., predicted minus the actual value, and their probability distribution. The difference/errors form an almost normal distribution, and most of them are between -2.5 and $2.5^{\circ} \mathrm{C}$. The result showed that $70 \%$ of the difference are less than $1^{\circ} \mathrm{C}$ and another $23 \%$ of the difference were less than $2{ }^{\circ} \mathrm{C}$. In other words, the model can forecast indoor temperature with a high accuracy, as $93 \%$ of the prediction could show a forecasting error within $2^{\circ} \mathrm{C}$.

Figure 5a illustrates the scores of selected features that correlated with indoor temperature. The feature score shows how close the linear relationship is between each of the input parameters and hourly indoor temperature; i.e., the output. Outdoor temperature and outdoor humidity have the highest impact on indoor temperature. Solar radiation contributed more than the rest of other input variables.

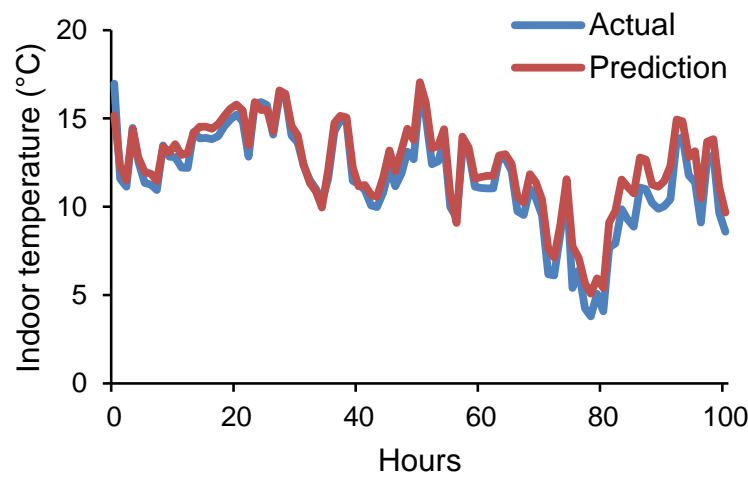

Figure 3: Predicted indoor temperatures using RNN model vs actual indoor temperatures over 100 hours.

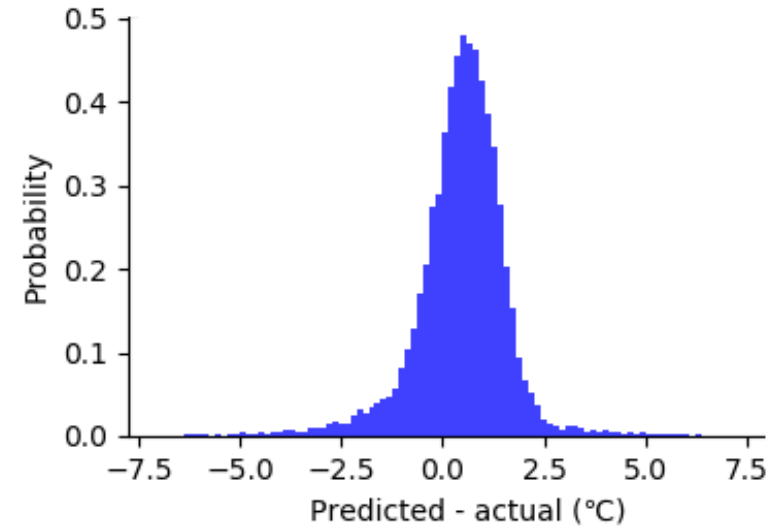

Figure 4: Difference between the predicted and actual indoor temperatures. $93 \%$ of the predicted values are within $2^{\circ} \mathrm{C}$ difference from the actual values.

Month, as an input variable, has a higher correlation with indoor temperature than other time-related inputs such as day and hour. The month can affect the indoor temperature more than day and hour as it is a proxy for seasons, and outdoor temperatures changes significantly between months. The feature score of window opening area is not as high as outdoor temperature and humidity; however, it is the most important factor following solar radiation and month.

Figure $5 \mathrm{~b}$ presents the feature score of each parameter for hourly change of indoor temperatures. The hourly solar radiation (both direct and diffuse) has the highest impact on the change in hourly indoor temperature. Following solar radiation, window opening area has the next highest feature score, showing high correlation between window opening and next-hour indoor temperature. Although window opening area could not perform as a good indicator for hourly indoor temperature, but it plays an important role in forecasting how (increase or decrease) and how much next-hour indoor temperature is going to change.

Figure 6 shows a positive trend between the outdoor and indoor temperatures. The linear regression model indicates an increase of $0.9387^{\circ} \mathrm{C}$ in indoor temperature when outdoor temperature increased $1^{\circ} \mathrm{C}$. The $\mathrm{R}^{2}$ of 0.8726 shows that there is a strong linear relationship between the variables. Therefore, the outdoor temperature could be used to predict indoor temperature. However, the linear equation only considers outdoor temperature. Through linear regression model on forecasting indoor temperatures, it needed to assess how accuracy (e.g., the percentage of $1^{\circ} \mathrm{C}$ error) this statistical method could forecast indoor temperatures. With this method, the proportion of $1{ }^{\circ} \mathrm{C}$ difference between the forecasted and actual temperatures was $50 \%$, which is considerably low compared to $70 \%$ with the LSTM-RNN method. The difference of $20 \%$ between the equation and RNN model was because it ignored other parameters such as humidity and window opening area. 


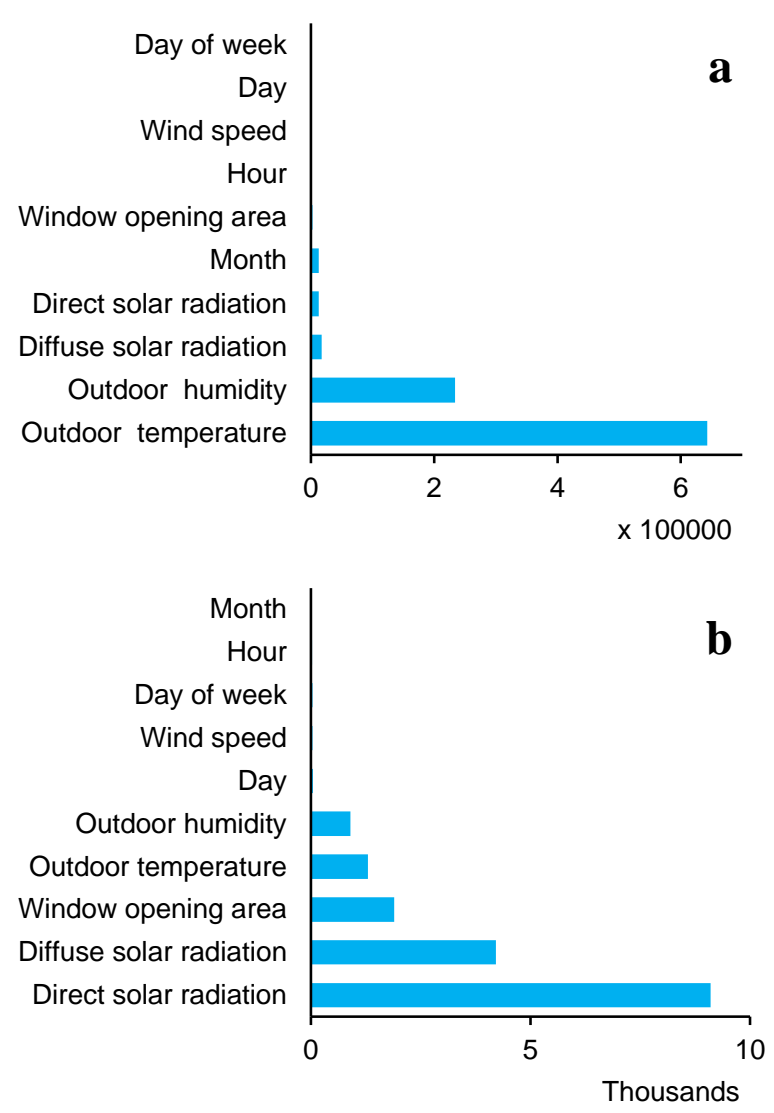

Feature score

Figure 5: Feature scores of input variables for different output variables. (a) Indoor temperature, and (b) hourly change in indoor temperature.

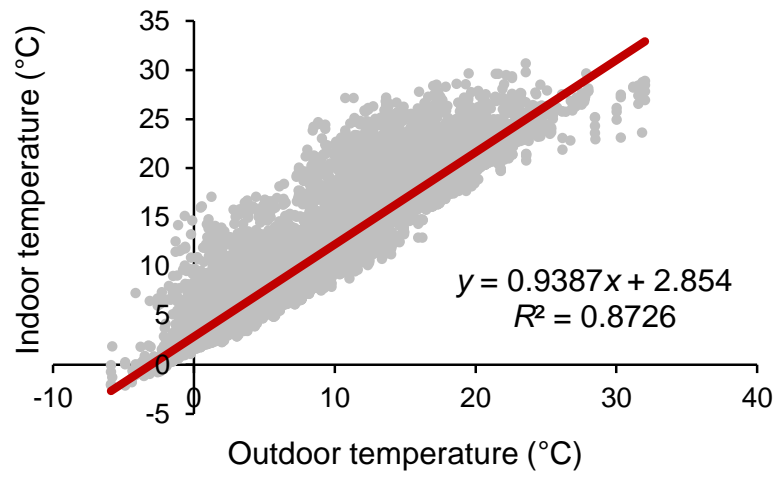

\section{Figure 6: Correlation between indoor and outdoor} temperatures.

Figure 7 illustrated the predicted indoor operative temperature based on the equation of the trend of the outdoor temperatures and indoor temperatures. Compared with prediction results from the RNN model, the prediction error was significantly greater. Overall, indoor temperatures were overestimated. However, due to the high correlation between outdoor temperatures and indoor temperatures, the regression model was capable of following the patterns of indoor temperature variation.

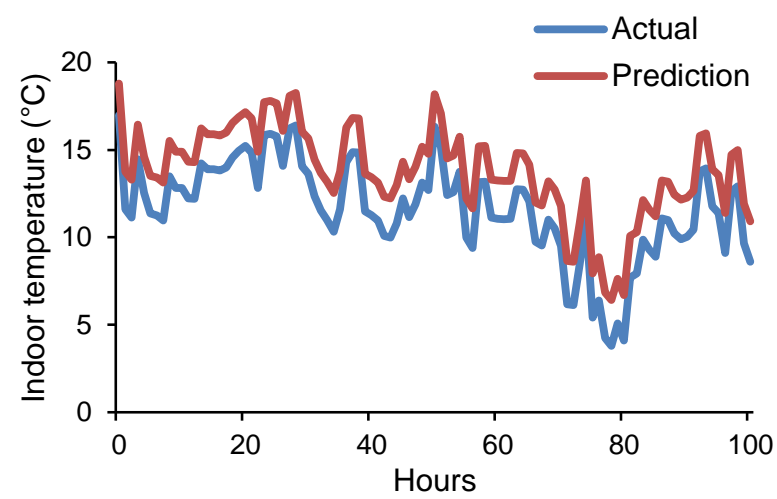

Figure 7: The prediction of indoor temperatures using regression over 100 hours.

\section{Conclusion}

In this paper, a data-driven machine learning algorithm LSTM-RNN is developed to forecast hourly indoor operative temperatures considering window opening area, time parameters and outdoor conditions. The results suggest that LSTM-RNN is an effective method in forecasting indoor temperatures. The model forecasted $70 \%$ of the hourly indoor temperatures with errors within $1^{\circ} \mathrm{C}$, and another $23 \%$ within $2^{\circ} \mathrm{C}$.

The research also demonstrates that indoor and outdoor temperatures are linearly correlated. Hypothetically, a regression model comprising only the outdoor and indoor temperatures can be used in lieu of an RNN model. However, the forecasting accuracy for the regression model was found to be inferior to the RNN model. Only $50 \%$ of the forecasted temperatures in a regression model are within $1{ }^{\circ} \mathrm{C}$ prediction error, compared to the $70 \%$ in the RNN model. While regression is able to follow the diurnal patterns of variation, indoor operative temperature is almost always overestimated.

Other than solar radiation, window opening area is the most important parameter that correlates highly with the hourly change of indoor temperatures. Hence, it is important in forecasting how and how much the next-hour or next-time-step indoor temperature will change.

The developed machine learning algorithm, LSTM-RNN can forecast the indoor temperature with high accuracy in seconds once the model has been trained and validated. Moreover, the data-driven approach also enables the prediction of indoor temperature on a low-power and compute-constrained hardware using window opening area as an input in a more reasonable time (seconds) than conventional computational fluid dynamics (CFD, needs hours) and network airflow models (minutes).

Further work can be conducted on near-real time control of building sub-systems (e.g., window opening automation) using LSTM-RNN. Due to its short-time response and high accuracy, near-real time optimal control of sub-systems in buildings for indoor thermal comfort and energy can be achieved. 


\section{Acknowledgement}

This work was partially funded by the European Commission via the Horizon 2020 project, TowArds Building rEady for Demand rEsponse (TABEDE) under Grant Agreement No. 766733.

\section{Reference}

Ahmad, M., Mourshed, M., Mundow, D., Sisinni, M., \& Rezgui, Y. (2016). Building energy metering and environmental monitoring - A state-of-the-art review and directions for future research. Energy and Buildings, 120, 85-102.

Artmann, N., Manz, H., \& Heiselberg, P. (2007). Climatic potential for passive cooling of buildings by nighttime ventilation in Europe. Applied energy, 84(2), 187-201.

Chen, Y., Norford, L., Samuelson, H., \& Malkawi, A. (2018). Optimal control of HVAC and window systems for natural ventilation through reinforcement learning. Energy and Buildings, 169, 195-205.

Chenari, B., Carrilho, J., \& da Silva, M. (2016). Towards sustainable, energy-efficient and healthy ventilation strategies in buildings: A review. Renewable and Sustainable Energy Reviews, 59, 1426-1447.

D'Oca, S., \& Hong, T. (2014). data-mining approach to discover patterns of window opening and closing behavior in offices. Building and Environment\}, 726739.

Giesekam, J., \& Pomponi, F. (2018). Briefing: Embodied carbon dioxide assessment in buildings: guidance and gaps. Proceedings of the ICE-Engineering Sustainability, 171(7), 334-341.

Grozinger, J., Boermans, T., Wehringer, A., \& Seehusen, J. (2014). Overview of Member States information on NZEBs: Background paper - Final Report. Cologne, Germany: ECOFYS GmbH.

Hamza, N., Zi, Q., \& Stein, O. (2015). User behaviours and preferences for low carbon homes: Lessons for predicting energy demand. Proceedings (p. 1). Hyderabad: International Buildinig Performance Simulation Association.

Lomas, K. (2007). Architectural design of an advanced naturally ventilated building form. Energy and Buildings, 39(2), 166-181.

Markovic, R., Frisch, J., \& van Treeck, C. (2019). Learning short-term past as predictor of window opening-related human behavior in commercial buildings. Energy and Buildings, 185, 1-11.

Marvuglia, A., Messineo, A., \& Nicolosi, G. (2014). Coupling a neural network temperature predictor and a fuzzy logic controller to perform thermal comfort regulation in an office building. Building and Environment, 72, 287-299.

Mourshed, M. (2011). The impact of the projected changes in temperature on heating and cooling requirements in buildings in Dhaka, Bangladesh. Applied Energy, 88(11), 3737-3746.

Peng, J., Curcija, D., Lu, L., Selkowitz, S., Yang, H., \& Zhang, W. (2016). Numerical investigation of the energy saving potential of a semi-transparent photovoltaic double-skin facade in a cool-summer Mediterranean climate. Applied Energy, 165, 345356.

Ramponi, R., Angelotti, A., \& Blocken, B. (2014). Energy saving potential of night ventilation: Sensitivity to pressure coefficients for different European climates. Applied Energy, 123, 185-195.

Ruiz, L., Cuellar, M., Calvo-Flores, M., \& Jimenez, M. (2016). An Application of Non-Linear Autoregressive Neural Networks to Predict Energy Consumption in Public Buildings. Energies, 9(9), 684.

Schulze, T., Gurlich, D., \& Eicker, U. (2018). Performance assessment of controlled natural ventilation for air quality control and passive cooling in existing and new office type buildings. Energy and Buildings, 172, 265-278.

Stocker, T. (2014). Climate change 2013: the physical science basis: Working Group I contribution to the Fifth assessment report of the Intergovernmental Panel on Climate Change. New York: Cambridge University Press.

Tong, Z., Chen, Y., Malkawi, A., Liu, Z., \& Freeman, R. (2016). Energy saving potential of natural ventilation in China: The impact of ambient air pollution. Applied energy, 179, 660-668.

Wang, Y., Velswamy, K., \& Huang, B. (2017). A LongShort Term Memory Recurrent Neural Network Based Reinforcement Learning Controller for Office Heating Ventilation and Air Conditioning Systems. Processes, 5(3), 46 .

Weng, K., Meng, F., \& Mourshed, M. (2018). ModelBased Optimal Control of Window Openings for Thermal Comfort. Proceedings, 2(15), 1134.

Xu, C., Chen, H., Wang, J., Guo, Y., \& Yuan, Y. (2019). Improving prediction performance for indoor temperature in public buildings based on a novel deep learning method. Building and Environment, 148, 128-135. 